\title{
A nomogram to predict the survival of stage IIIA-N2 non-small cell lung cancer after surgery
}

Qixing Mao, MD, ${ }^{\text {a,b,c }}$ Wenjie Xia, MD, ${ }^{\mathrm{a}, \mathrm{b}, \mathrm{c}}$ Gaochao Dong, MS, ${ }^{\mathrm{a}, \mathrm{c}}$ Shuqi Chen, PhD, ${ }^{\mathrm{e}}$

Anpeng Wang, MD, ${ }^{\mathrm{a}, \mathrm{b}, \mathrm{c}}$ Guangfu Jin, $\mathrm{PhD},{ }^{\mathrm{d}}$ Feng Jiang, $\mathrm{MD}, \mathrm{PhD},{ }^{\mathrm{a}, \mathrm{c}}$ and $\mathrm{Lin} \mathrm{Xu}, \mathrm{MD}, \mathrm{PhD}^{\mathrm{a}, \mathrm{c}}$

\section{ABSTRACT}

Objective: Postoperative survival of patients with stage IIIA-N2 non-small cell lung cancer (NSCLC) is highly heterogeneous. Here, we aimed to identify variables associated with postoperative survival and develop a tool for survival prediction.

Methods: A retrospective review was performed in the Surveillance, Epidemiology, and End Results database from January 2004 to December 2009. Significant variables were selected by use of the backward stepwise method. The nomogram was constructed with multivariable Cox regression. The model's performance was evaluated by concordance index and calibration curve. The model was validated via an independent cohort from the Jiangsu Cancer Hospital Lung Cancer Center.

Results: A total of 1809 patients with stage IIIA-N2 NSCLC who underwent surgery were included in the training cohort. Age, sex, grade, histology, tumor size, visceral pleural invasion, positive lymph nodes, lymph nodes examined, and surgery type (lobectomy vs pneumonectomy) were identified as significant prognostic variables using backward stepwise method. A nomogram was developed from the training cohort and validated using an independent Chinese cohort. The concordance index of the model was 0.673 (95\% confidence interval, $0.654-0.692)$ in training cohort and 0.664 in validation cohort $(95 \%$ confidence interval, 0.614-0.714). The calibration plot showed optimal consistency between nomogram predicted survival and observed survival. Survival analyses demonstrated significant differences between different subgroups stratified by prognostic scores.

Conclusions: This nomogram provided the individual survival prediction for patients with stage IIIA-N2 NSCLC after surgery, which might benefit survival counseling for patients and clinicians, clinical trial design and follow-up, as well as postoperative strategy-making. (J Thorac Cardiovasc Surg 2018;155:1784-92)

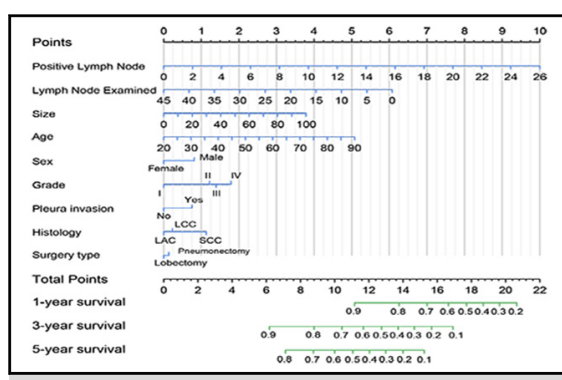

Nomogram estimates survival probabilities by adding up scores of all risk variables.

\section{Central Message}

We developed a nomogram to predict the postoperative survival of stage IIIA-N2 lung cancer, which would improve the individualized survival prediction and assist the postoperative strategy-making.

\section{Perspective}

Postoperative survival of patients with stage IIIA-N2 lung cancer is heterogeneous. The nomogram was developed by significant variables related to survival from an US dataset and validated in a Chinese cohort. It might benefit survival counseling, clinical trial design, as well as postoperative strategymaking. Meanwhile, it would drive the development of precise medicine.

See Editorial Commentary page 1793

See Editorial page 1783.
Stage IIIA-N2 non-small cell lung cancer (NSCLC) accounts for approximately $15 \%$ of NSCLC. The management of this stage is the most controversial issue in the area of lung cancer. ${ }^{1-3}$ The main reason is that the patients

From the ${ }^{\text {aDepartment }}$ of Thoracic Surgery, Jiangsu Cancer Hospital, Jiangsu Institute of Cancer Research, Nanjing Medical University Affiliated Cancer Hospital, Nanjing, China; ' The Fourth Clinical College of Nanjing Medical University, Nanjing, China; ' Jiangsu Key Laboratory of Molecular and Translational Cancer Research, Nanjing Medical University Affiliated Cancer Hospital, Cancer Institute of Jiangsu Province, Nanjing, China; ${ }^{\mathrm{d}}$ Department of Epidemiology and Biostatistics, Jiangsu Key Lab of Cancer Biomarkers, Prevention and Treatment, Collaborative Innovation Center for Cancer Medicine, School of Public Health, Nanjing Medical University, Nanjing, China; and ${ }^{\mathrm{e}}$ Department of Health Statistics, Second Military Medical University, Shanghai, China.

This research was supported by the National Natural Science Foundation of China (nos. 81472702, 81501977, and 81672294), Natural Science Foundation of Jiangsu with stage IIIA-N2 NSCLC are heterogeneous in distinct tumor extension and involvement of mediastinal lymph nodes. ${ }^{4}$ Patients with single-station or microscopic disease had better postoperative survival than those with

Province (no. SBK016030028), and the Innovation Capability Development Project of Jiangsu Province (no. BM2015004).

Drs Qixing and Wenjie and Mr Gaochao contributed equally to this article.

Received for publication April 19, 2017; revisions received Nov 9, 2017; accepted for publication Nov 16, 2017.

Address for reprints: Lin $\mathrm{Xu}, \mathrm{MD}$, PhD, or Feng Jiang, MD, PhD, Baiziting 42,

Nanjing, China, 210009 (E-mail: xulin_83@hotmail.com or zengnljf@hotmail. com).

$0022-5223 / \$ 36.00$

Copyright (C) 2017 by The American Association for Thoracic Surgery

https://doi.org/10.1016/j.jtcvs.2017.11.098 


$$
\begin{aligned}
& \text { Abbreviations and Acronyms } \\
& \begin{aligned}
\text { CI } & =\text { confidence interval } \\
\text { HR } & =\text { hazard ratio } \\
\text { NSCLC } & =\text { non-small cell lung cancer } \\
\text { OS } \quad & \text { overall survival } \\
\text { SEER } & \text { Surveillance, Epidemiology, and End } \\
& \text { Results }
\end{aligned}
\end{aligned}
$$

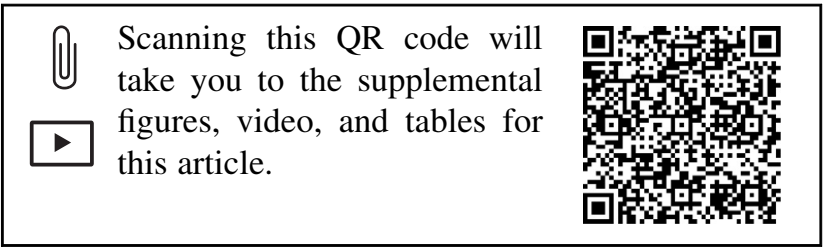

multistation or bulky nodal disease, shown as a 5-year survival rate of $34 \%$ and $3 \%$, respectively. ${ }^{5,6}$ In addition, the heterogeneity also comes from physical condition of individuals, including age, sex, and performance status, which all play critical roles in prognosis. ${ }^{4}$ Furthermore, the optimal treatment for stage IIIA-N2 NSCLC remains a matter of debate. ${ }^{7,8}$ Different therapeutic strategies result in diverse survival. Thus, the aforementioned heterogeneities raise uncertainties and difficulties in risk stratification and postoperative survival prediction, which impede the development of precise medicine. Although several substage approaches were implemented by stratifying patients into more accurate subgroups, a postoperative survival prediction model is in urgent need to develop precise medicine for patients with stage IIIAN2 NSCLC.

A nomogram is a feasible tool to describe the individual prognosis or risk of a clinical event. ${ }^{9}$ By this model, clinicians can easily obtain the survival of an individual by a simple graphic. ${ }^{10}$ Several nomograms regarding NSCLC were published. ${ }^{11,12}$ However, few studies have addressed the prognosis of locally advanced NSCLC. Thus, our study aimed to establish a nomogram to predict the postoperative survival for patients with stage IIIA-N2 NSCLC using a cohort from the Surveillance, Epidemiology, and End Results (SEER) database and validate it in an independent cohort from Jiangsu Cancer Hospital Lung Cancer Center.

\section{METHODS}

\section{Study Population}

The training cohort of this nomogram was derived from SEER, which is a population-based database and covers approximately $28 \%$ of the American population. The latest data, released in April 2016, were downloaded from SEER. It enrolled patients with stage IIIA-N2 NSCLC who underwent surgery from January 2004 to December 2009 and excluded those younger than 18 years old. The staging method was defined according to the 7th American Joint Committee on Cancer-Tumor, Node, and Metastasis staging system. The common histologic types were included as follows: large cell carcinoma $(8012-8014,8310,8123,8082)$, squamous cell carcinoma (8052, 8070-8074, 8083-8084), and adenocarcinoma (8123, 8140, $8230,8250-8255,8260,8310,8333,8470,8480-8481,8490,8550)$. The tumor grade was classified into 4 levels, including well-differentiated, moderately differentiated, poorly differentiated, and undifferentiated. The codes of tumor sites were restricted from C34.0 to C34.9, ranging from the main bronchus to each lobe. Patients who underwent lobectomy, bilobectomy, or pneumonectomy by either a thoracotomy or video-assisted thoracic surgery were included. In addition, we categorized surgery type into 2 groups: lobectomy and pneumonectomy. Patients who received radiation therapy were also enrolled in the cohort. Therapy type was classified based on sequence of radiation therapy and surgery: radiation therapy before surgery, radiation therapy after surgery, and surgery alone. Other specific criteria for selection were as follows: (1) tumor extension (codes: 100-800), (2) number of lymph nodes examined (codes: 1-90), and (3) number of regional lymph nodes positive (codes: 0-90). According to tumor extension, we divided patients into visceral pleural invasion and no visceral pleural invasion. The aforementioned procedures were performed by SEER* Stat Version 8.3.2 (National Cancer Institute, Bethesda, Md), released in April 2016. We also excluded patients with unknown lymph node status, unknown grade, and without pathologic diagnosis. Finally, age, sex, tumor site, tumor size, histology, grade, lymph nodes examined, positive lymph nodes, surgery type, therapy type, and visceral pleural invasion were incorporated into our study.

An independent cohort was constructed from the Department of Thoracic Surgery Lung Cancer Center, Jiangsu Cancer Hospital, from 2003 to 2011. Patients were selected by the same criteria used in the training cohort. Ethical approval was given by the Nanjing Medical University Ethics Committee.

The primary outcome was defined as overall survival (OS). Time of OS was counted from the date of diagnosis to the date of death or last contact. The SEER included dates of last contact up to December 31, 2013. The last contact of the validation cohort was up to December 2016.

\section{Constructing the Nomogram}

Continuous variables were summarized as median values with interquartile range. Median survival time with $95 \%$ confidence interval (CI) was calculated by the Kaplan-Meier method. The development of the prognostic model started with a univariate assessment of the effect of each clinicopathologic parameter with a Cox proportional hazards regression model. Univariate hazard ratio (HR) and $P$ values were calculated. Significant variables were selected by the backward stepwise selection method based on Akaike information criterion. ${ }^{13}$ Variables no longer contributing either statistically or clinically on statistical adjustment were then excluded. Based on the significant variables, a nomogram was constructed via the RMS package in R 3.3.1 (R Foundation for Statistical Computing, Vienna, Austria). ${ }^{10}$ The Cox proportional hazard model was selected. ${ }^{14}$

\section{VATS Left Upper Lobectomy}

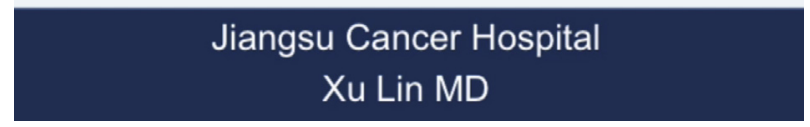

VIDEO 1. This study constructed a nomogram to predict the postoperative survival for patients with stage IIIA-N2 NSCLC. This video shows the surgical procedures for a patient with stage IIIA-N2 NSCLC. NSCLC, Non-small cell lung cancer. Video available at: http://www.jtcvsonline. org/article/S0022-5223(17)33004-0/fulltext. 
TABLE 1. Demographic and pathologic variables of training and validation cohort

\begin{tabular}{|c|c|c|c|c|c|c|c|c|}
\hline \multirow[b]{3}{*}{ Demographic } & \multicolumn{4}{|c|}{ Training cohort } & \multicolumn{4}{|c|}{ Validation cohort } \\
\hline & \multirow[b]{2}{*}{ No. patients } & \multirow[b]{2}{*}{$\%$} & \multicolumn{2}{|c|}{ Overall survival, mo } & \multirow[b]{2}{*}{ No. patients } & \multirow[b]{2}{*}{$\%$} & \multicolumn{2}{|c|}{ Overall survival, mo } \\
\hline & & & Median & $95 \%$ CI & & & Median & $95 \% \mathrm{CI}$ \\
\hline Age, y & \multicolumn{4}{|c|}{1809} & \multicolumn{4}{|c|}{124} \\
\hline Age, y, median (IQR) & $65(57.5-72.5)$ & - & - & - & $61(54-68)$ & - & - & - \\
\hline \multicolumn{9}{|l|}{ Age groups, y } \\
\hline $20-30$ & 2 & 0.1 & 59.0 & - & - & - & - & - \\
\hline $31-40$ & 19 & 1.1 & 69.0 & $52.1-84.3$ & 4 & 3.2 & 16.8 & 7.6-25.9 \\
\hline $41-50$ & 141 & 7.8 & 53.0 & $39.8-66.2$ & 18 & 14.5 & 22.3 & $18.7-26.0$ \\
\hline $51-60$ & 416 & 23.0 & 43.0 & $35.8-50.2$ & 41 & 33.1 & 32.7 & $14.2-51.3$ \\
\hline $61-70$ & 620 & 34.3 & 39.0 & $34.7-43.3$ & 35 & 28.2 & 29.8 & $20.2-41.8$ \\
\hline $71-80$ & 502 & 27.7 & 31.0 & $26.5-35.5$ & 26 & 21.0 & 24.8 & $11.9-34.9$ \\
\hline $81-90$ & 109 & 6.0 & 29.7 & $20.0-34.9$ & - & - & - & - \\
\hline \multicolumn{9}{|l|}{ Sex } \\
\hline Female & 950 & 52.5 & 44.0 & $39.6-48.3$ & 39 & 31.5 & 38.5 & $13.7-63.3$ \\
\hline Male & 859 & 47.5 & 30.0 & $26.4-33.6$ & 85 & 68.5 & 34.5 & $18.1-50.8$ \\
\hline \multicolumn{9}{|l|}{ Tumor site } \\
\hline Main bronchus & 22 & 1.2 & 36.0 & $16.5-55.5$ & - & - & - & - \\
\hline Upper lobe & 1043 & 57.7 & 40.0 & $35.9-44.0$ & - & - & - & - \\
\hline Middle lobe & 84 & 4.6 & 42.0 & $33.0-50.9$ & - & - & - & - \\
\hline Lower lobe & 602 & 33.3 & 34.0 & $30.1-37.9$ & - & - & - & - \\
\hline Overlap lobe & 39 & 2.2 & 28.0 & $15.6-40.4$ & - & - & - & - \\
\hline Lung, NOS & 19 & 1.0 & 18.0 & $0.9-35.0$ & - & - & - & - \\
\hline \multicolumn{9}{|l|}{ Histology } \\
\hline Adenocarcinoma & 1294 & 71.5 & 40.0 & $36.6-43.4$ & 78 & 62.9 & 42.1 & $24.0-60.2$ \\
\hline Lung squamous cell carcinoma & 444 & 24.5 & 30.0 & $24.0-33.9$ & 46 & 37.1 & 34.5 & $12.4-56.6$ \\
\hline Large cell carcinoma & 71 & 3.9 & 33.0 & $20.6-45.3$ & - & - & - & - \\
\hline \multicolumn{9}{|l|}{ Grade } \\
\hline Well-differentiated & 96 & 5.3 & 58.0 & $42.5-71.6$ & 6 & 4.8 & 42.1 & $35.2-48.5$ \\
\hline Moderately differentiated & 803 & 44.4 & 41.0 & $36.8-45.2$ & 43 & 34.7 & 38.5 & $22.8-54.2$ \\
\hline Poorly differentiated & 847 & 46.9 & 33.0 & $29.0-36.9$ & 55 & 44.4 & 25.4 & $12.8-38.0$ \\
\hline Undifferentiated & 63 & 3.4 & 23.0 & $15.3-30.7$ & 20 & 16.1 & 25.1 & $10.5-36.6$ \\
\hline \multicolumn{9}{|l|}{ Lymph nodes examined, number } \\
\hline Median & 10 & - & - & - & 8 & - & - & - \\
\hline IQR & $6-15$ & - & - & - & $11-15$ & - & - & - \\
\hline $0-10$ & 985 & 54.4 & 34.0 & $30.8-37.2$ & 60 & 48.4 & 40.4 & $23.8-57.0$ \\
\hline $11-20$ & 586 & 32.4 & 40.0 & $34.8-45.2$ & 56 & 45.2 & 34.5 & $8.8-60.2$ \\
\hline $21-30$ & 173 & 9.6 & 47.0 & $38.3-55.7$ & 8 & 6.5 & 38.5 & $23.9-56.8$ \\
\hline $31-45$ & 65 & 3.6 & 39.0 & $19.0-58.9$ & - & - & - & - \\
\hline \multicolumn{9}{|l|}{ Positive lymph nodes, number } \\
\hline Median & 3 & - & - & - & 3 & - & - & - \\
\hline IQR & $1-5$ & - & - & - & $1-5$ & - & - & - \\
\hline $0-5$ & 1475 & 81.5 & 41.0 & $37.6-44.4$ & 100 & 80.6 & 43.4 & $27.6-59.3$ \\
\hline $6-10$ & 252 & 13.9 & 28.0 & $23.9-32.1$ & 21 & 16.9 & 21.7 & $18.2-25.4$ \\
\hline $11-15$ & 58 & 3.2 & 22.0 & $13.3-30.7$ & 3 & 2.4 & 15.6 & $11.2-19.3$ \\
\hline $16-20$ & 13 & 0.7 & 18.0 & $10.0-26.0$ & - & - & - & - \\
\hline $21-26$ & 11 & 0.6 & 9.0 & $3.6-14.4$ & - & - & - & - \\
\hline Tumor size, mm & & & & & & & & \\
\hline Median & 34 & - & - & - & 50 & - & - & - \\
\hline IQR & $23-49$ & - & - & - & $30-72$ & - & - & - \\
\hline $0-20$ & 363 & 20.1 & 62.0 & $53.2-70.8$ & 5 & 4.0 & 52.7 & $34.3-71.2$ \\
\hline $21-30$ & 453 & 25.0 & 43.0 & $36.6-49.4$ & 35 & 28.2 & 43.5 & $20.7-66.2$ \\
\hline $31-50$ & 603 & 33.3 & 33.0 & 29.1-36.9 & 24 & 20.2 & 24.3 & $14.3-35.4$ \\
\hline
\end{tabular}


TABLE 1. Continued

\begin{tabular}{|c|c|c|c|c|c|c|c|c|}
\hline \multirow[b]{3}{*}{ Demographic } & \multicolumn{4}{|c|}{ Training cohort } & \multicolumn{4}{|c|}{ Validation cohort } \\
\hline & \multirow[b]{2}{*}{ No. patients } & \multirow[b]{2}{*}{$\%$} & \multicolumn{2}{|c|}{ Overall survival, mo } & \multirow[b]{2}{*}{ No. patients } & \multirow[b]{2}{*}{$\%$} & \multicolumn{2}{|c|}{ Overall survival, mo } \\
\hline & & & Median & 95\% CI & & & Median & $95 \%$ CI \\
\hline $51-70$ & 259 & 14.3 & 26.0 & $20.6-31.4$ & 27 & 21.8 & 23.7 & $12.7-34.8$ \\
\hline$>71$ & 131 & 7.2 & 19.0 & $15.8-22.3$ & 33 & 25.8 & 22 & $1.2-27.8$ \\
\hline \multicolumn{9}{|l|}{ Surgery type } \\
\hline Lobectomy & 1602 & 88.7 & 39.0 & $35.9-42.0$ & 113 & 91.1 & 34.7 & $14.5-48.3$ \\
\hline Pneumonectomy & 207 & 11.3 & 28.0 & $23.7-33.3$ & 11 & 8.9 & 19.9 & $14.6-25.1$ \\
\hline \multicolumn{9}{|l|}{ Therapy } \\
\hline Surgery only & 1139 & 62.9 & 37.0 & $33.8-40.2$ & 110 & 88.7 & 19.1 & $15.2-22.2$ \\
\hline Radiation after surgery & 558 & 30.8 & 37.0 & $32.3-42.7$ & 10 & 8.1 & 14.43 & $8.9-19.9$ \\
\hline Radiation before surgery & 112 & 6.3 & 42.0 & $35.6-58.4$ & 4 & 3.2 & 42.1 & $23.1-52.9$ \\
\hline \multicolumn{9}{|l|}{ Visceral pleural invasion } \\
\hline No & 879 & 48.6 & 45.0 & $39.7-50.3$ & 65 & 52.4 & 54.4 & $36.8-74.7$ \\
\hline Yes & 930 & 51.4 & 31.0 & 27.9-34.1 & 59 & 47.6 & 26.2 & 23.4-28.9 \\
\hline
\end{tabular}

$C I$, Confidence interval; $I Q R$, interquartile range; $N O S$, not otherwise specified.

\section{Validation and Calibration}

This model was subject to 1000 bootstrap resamples for internal validation. External validation was performed with an independent cohort. Concordance index (C-index) and its 95\% CIs were calculated to evaluate the performance of the nomogram. ${ }^{15}$ The value of the $\mathrm{C}$-index ranged from 0.5 to 1.0 , with 0.5 implying no discrimination and 1.0 indicating a perfect prediction. A calibration plot described the fitting degree between the actual survival and the nomogram predicting survival. In addition, we divided patients into different risk groups according to prognostic scores. Log-rank survival analysis was used to identify the different survival between different risk groups to test the discriminate ability of the nomogram. The optimal cut-off values of the subgroups were defined by X-tile software (http://medicine.yale.edu/lab/rimm/research/software. aspx). ${ }^{16}$ The software could rationally divide continuous variable into several subsets with the largest $\chi^{2} \log$-rank value and the minimum $P$ value.

\section{Statistical Analysis}

Statistical analysis was performed by SPSS software, version 21.0 (SPSS Inc, Armonk, NY). Cox regression analysis was applied to identify the variables related to survival. HRs with $95 \%$ CIs were calculated. Kaplan-Meier curves were used to estimate distinct prognosis by the log-rank test.

\section{RESULTS}

\section{Demographics and Clinicopathologic Characteristics}

A total of 1809 patients were collected from the SEER database for the training cohort (Figure E1). One hundred twenty-four patients were retrospectively identified from the Lung Cancer Center in Jiangsu Cancer Hospital for validation (see surgical procedures for stage IIIA-N2 NSCLC in Video 1). Median of age at diagnosis in the training and validation cohorts were 66 and 60 years old, respectively. The proportion of male patients was close to that of female patients in both cohorts. Upper lobes were the most common primary tumor site compared with other sites. More than $60 \%$ of patients were diagnosed with adenocarcinoma. Lobectomy occupied a major proportion in both cohorts. Nearly $10 \%$ of patients underwent preoperative radiation therapy, and one third of patients received postoperative radiation therapy. The median follow-up duration was 39 months in the training cohort and 24 months in the validation cohort. During the follow-up period, 1284 (71.0\%) patients in the training group died and $65(52.4 \%)$ patients in the validation group died. Baseline information with median survival time is listed in Table 1.

\section{Selecting Potential Variables}

Variables including age, sex, histology, grade, lymph node examined, positive lymph node, visceral pleural invasion, tumor size, therapy type, and surgery type were significantly correlated with postoperative survival of stage IIIAN2 NSCLC by Cox analyses (Table 2). Male sex and visceral pleural invasion were identified as independent risk factors by multivariate cox analysis. Surgery type was not significant when adjusted with other variables. Patients with squamous cell carcinoma showed a greater HR than those with adenocarcinoma $(P=.040)$, whereas no significant difference was found between large cell carcinoma and adenocarcinoma $(P=.375)$. With respect to tumor grade, we found that moderate grade, poor grade, and undifferentiated grade were prognostic indicators compared with the well-differentiated grade $(P=.013, P=.005$, and $P=.021$, respectively). As the grade increased, survival time decreased. Age, tumor size, and number of positive lymph nodes were also independent prognostic factors. The number of lymph nodes examined partly reflected the extension of lymphadenectomy. The number of lymph nodes examined showed a protective effect to survival $(P<.001)$. Variables were then selected to develop a nomogram by the backward stepwise analysis.

\section{Developing the Prognostic Nomogram}

A nomogram was established by significant variables to estimate the probability of OS in the training cohort (Figure 1). In the nomogram, each variable was assigned 
TABLE 2. Univariable and multivariable Cox proportional hazards analysis

\begin{tabular}{|c|c|c|c|c|c|c|}
\hline \multirow[b]{2}{*}{ Variable } & \multicolumn{2}{|c|}{ Univariable analysis } & \multicolumn{2}{|c|}{ Multivariable analysis } & \multicolumn{2}{|c|}{ Selected factors for building the model } \\
\hline & Hazard ratio $(95 \%$ CI $)$ & $P$ value & $\overline{\text { Hazard ratio }(95 \% \mathrm{CI})}$ & $P$ value & Hazard ratio $(95 \% \mathrm{CI})$ & $P$ value \\
\hline Age, y & $1.021(1.015-1.026)$ & $<.001^{*}$ & $1.020(1.014-1.026)$ & $<.001 *$ & $1.020(1.014-1.026)$ & $<.001 *$ \\
\hline \multicolumn{7}{|l|}{ Sex } \\
\hline Female & Ref & $<.001 *$ & Ref & $<.001 *$ & Ref & $<.001 *$ \\
\hline Male & $1.340(1.202-1.471)$ & & $1.256(1.122-1.407)$ & & $1.263(1.127-1.415)$ & \\
\hline \multicolumn{7}{|l|}{ Tumor site } \\
\hline Main bronchus & Ref & .185 & - & - & - & - \\
\hline Upper lobe & $0.856(0.427-1.714)$ & 660 & - & - & - & - \\
\hline Middle lobe & $0.697(0.418-1.162)$ & .167 & - & - & - & - \\
\hline Lower lobe & $0.665(0.374-1.181)$ & .164 & - & - & - & - \\
\hline Overlap lobe & $0.790(0.472-1.321)$ & .368 & - & - & - & - \\
\hline Lung, NOS & $0.872(0.467-1.626)$ & 666 & - & - & - & - \\
\hline \multicolumn{7}{|l|}{ Histology } \\
\hline Adenocarcinoma & Ref & $.028^{*}$ & Ref & $.102 *$ & Ref & $.060^{*}$ \\
\hline Lung squamous cell carcinoma & $1.164(1.026-1.320)$ & .018 & $1.398(1.015-1.923)$ & .040 & $1.413(1.027-1.945)$ & .034 \\
\hline Large cell carcinoma & $1.014(0.944-1.113)$ & .124 & $1.064(0.928-1.219)$ & .375 & $1.099(0.960-1.258)$ & .172 \\
\hline \multicolumn{7}{|l|}{ Grade } \\
\hline Well-differentiated & Ref & $.001^{*}$ & Ref & $.016^{*}$ & Ref & $.0014^{*}$ \\
\hline Moderately differentiated & $1.235(0.940-1.621)$ & .129 & $1.409(1.073-1.849)$ & .013 & $1.389(1.059-1.823)$ & .018 \\
\hline Poorly differentiated & $1.375(1.048-1.804)$ & .021 & $1.481(1.128-1.946)$ & .005 & $1.455(1.108-1.912)$ & .007 \\
\hline Undifferentiated & $1.784(1.222-2.603)$ & .003 & $1.638(1.076-2.492)$ & .021 & $1.655(1.088-2.517)$ & .019 \\
\hline Lymph nodes examined, number & $0.990(0.983-0.997)$ & $<.001^{*}$ & $0.965(0.957-0.973)$ & $<.001^{*}$ & $0.965(0.957-0.973)$ & $<.001 *$ \\
\hline Positive lymph nodes, number & $1.069(1.054-1.084)$ & $<.001 *$ & $1.104(1.086-1.122)$ & $<.001 *$ & $1.104(1.086-1.123)$ & $<.001 *$ \\
\hline Tumor size, mm & $1.010(1.008-1.012)$ & $<.001^{*}$ & $1.011(1.008-1.014)$ & $<.001 *$ & $1.008(1.006-1.011)$ & $<.001 *$ \\
\hline \multicolumn{7}{|l|}{ Visceral pleural invasion } \\
\hline Yes & Ref & $<.001 *$ & Ref & $<.001 *$ & Ref & $<.001 *$ \\
\hline No & $0.839(0.795-0.887)$ & $<.001$ & $0.809(0.720-0.909)$ & $<.001$ & $0.797(0.709-0.895)$ & $<.001$ \\
\hline \multicolumn{7}{|l|}{ Surgery type } \\
\hline Lobectomy & Ref & $.001 *$ & Ref & $.149^{*}$ & Ref & $.15^{*}$ \\
\hline Pneumonectomy & $1.309(1.113-1.540)$ & & $1.025(0.853-1.231)$ & & $1.025(0.853-1.231)$ & \\
\hline \multicolumn{7}{|l|}{ Therapy } \\
\hline Surgery only & Ref & .129 & Ref & .316 & - & - \\
\hline Radiation before surgery & $1.074(0.975-1.184)$ & .147 & $1.014(0.824-1.132)$ & .131 & - & - \\
\hline Radiation after surgery & $0.834(0.656-1.030)$ & .073 & $0.857(0.683-1.106)$ & .200 & - & - \\
\hline
\end{tabular}

CI, Confidence interval; Ref, reference; NOS, not otherwise specified. *Statistically significant.

to a point ranging from 0 to 10 , which was converted by its coefficient in Cox regression model. The model equation and coefficients of variables were showed in Table E1. We found that the number of positive lymph nodes made the largest contribution to prognosis and was assigned to a point of 10. A reverse relationship was observed between the number of lymph nodes examined with its corresponding point. Grade, tumor size, and age showed a moderate impact on the prognosis. The total points for each patient, ranging from 0 to 21.9 in the training cohort, was added by points of all variables. When drawing a vertical line from total points scale to survival scales, we could individually estimate the probabilities of survival at 1 year, 3 years, and 5 years. Probabilities of survival at
1 year, 3 years, and 5 years ranged from 0.2 to $0.9,0.1$ to 0.9 , and 0.1 to 0.8 , respectively.

\section{Calibration and Validation of the Nomogram}

The c-statistic for the Cox regression model was 0.671 (95\% CI, 0.653-0.689) in the training set and $0.666(95 \%$ CI, 0.608-0.724) in validation set. The C-index, evaluating the performance of the nomogram, was 0.673 in training cohort (95\% CI, 0.654-0.692). In the validation cohort, the C-index was 0.664 (95\% CI, 0.614-0.714), which was close to that in the training cohort. A calibration plot presented optimal prediction for 3-year and 5-year OS in the training cohort by 330-sample bootstrapped (Figure 2). The calibration plot of the external validation cohort also 


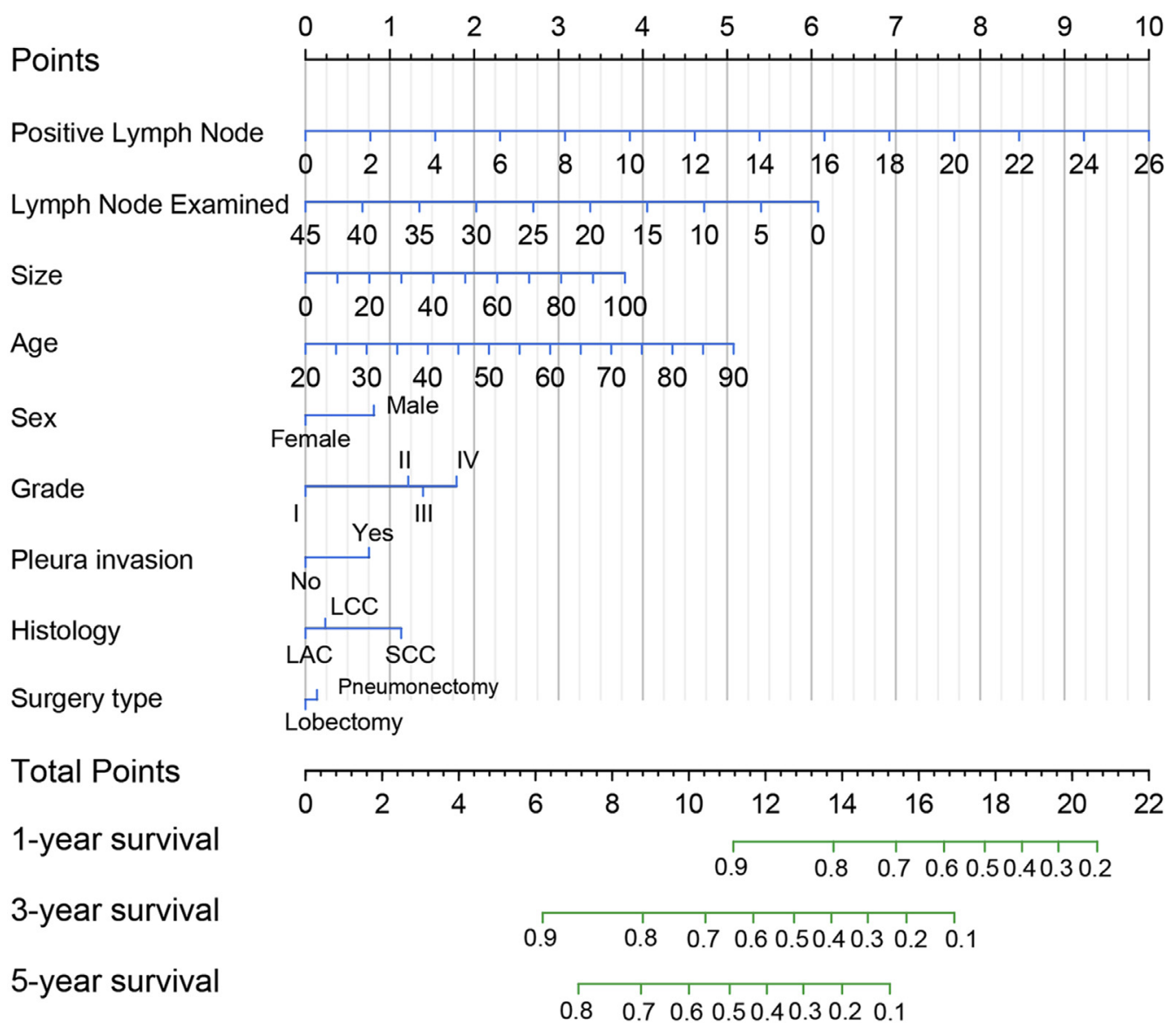

FIGURE 1. Nomogram predicting postoperative survival of patients with stage IIIA-N2 non-small cell lung cancer. The units of continuous variables are as follows: no. of positive lymph nodes, no. lymph nodes examined, size in millimeters, and age in years. I, well differentiated, II, moderately differentiated, III, poorly differentiated, IV, undifferentiated. $L C C$, Large cell carcinoma, $L A C$, lung adenocarcinoma, $S C C$, squamous cell carcinoma.

provided an accordant agreement between nomogram prediction and actual observation (Figure 3).

\section{Discriminative Ability of the Nomogram}

We divided patients into 4 risk subgroups with the largest $\chi^{2}$ log-rank value 32.4 using $\mathrm{X}$-tile software $(P<.0001)$. The optimal cut-off values for total points were classified as follows: 0-12.5, 12.6-13.6, 13.7-16.4, and 16.5-21.9. Survival analyses demonstrated significant distinctions between subgroups in the training cohort (Figure 4). The same procedures were performed in the validation cohort, and survival differences were observed among subgroups (Figure 5). The $\log$-rank $P$ value of each subgroup is listed in Table 3.

\section{DISCUSSION}

Stage IIIA-N2 NSCLC represents a heterogeneous group of patients with diverse survival. This was the first attempt to establish a prediction model for long-term survival of resectable patients with locally advanced NSCLC. It was developed from a large dataset in the United States and validated by an independent cohort of Chinese patients. By incorporating 9 preoperative and intraoperative variables, the current nomogram could benefit survival counseling for patients and clinicians, clinical trial design, and follow-up, as well as postoperative strategy-making, which might drive development of precise medicine.

There are several published nomograms concerning survival prediction for NSCLC. Liang and colleagues ${ }^{11}$ developed and validated a nomogram that could provide an individual prediction of OS for patients with early-stage NSCLC. Jin and colleagues ${ }^{17}$ established a nomogram that could predict the risk of invasive pulmonary adenocarcinoma for patients with solitary peripheral subsolid nodules. In addition, Zhang and colleagues ${ }^{12}$ reported a clinicopathologic prediction model for postoperative recurrence in early stage NSCLC. However, previous models were primarily aimed at early-stage NSCLC and excluded the patients receiving neoadjuvant or adjuvant therapy, which were not fit for survival prediction for stage IIIA$\mathrm{N} 2$ patients. In our nomogram, we enrolled all resectable patients with stageIIIA-N2 and didn't exclude those 


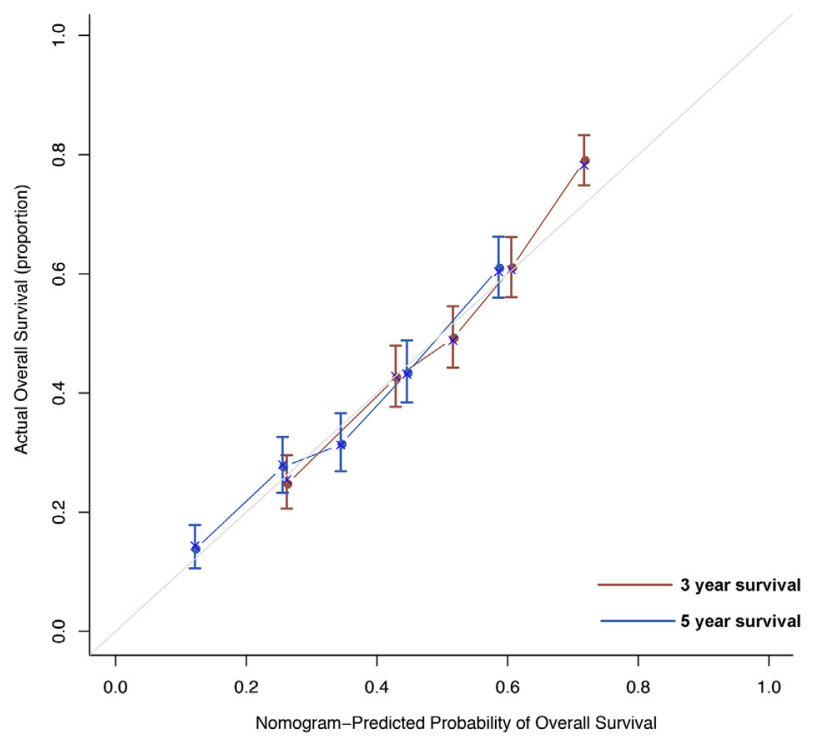

FIGURE 2. The calibration plot for the nomogram in the training cohort. Nomogram-predicted survival is plotted on the $\mathrm{x}$-axis; observation survival is plotted on the y-axis. A plot along the 45-degree line indicated a perfect calibration model in which the predicted probabilities were consistent with the actual outcomes. The blue line represents 5-year survival. The red line represents 3-year survival.

receiving neoadjuvant or adjuvant therapy, which could maintain the universality of the model. In addition, a comprehensive panel of clinicopathologic variables was adopted to guarantee the accuracy and reliability of the

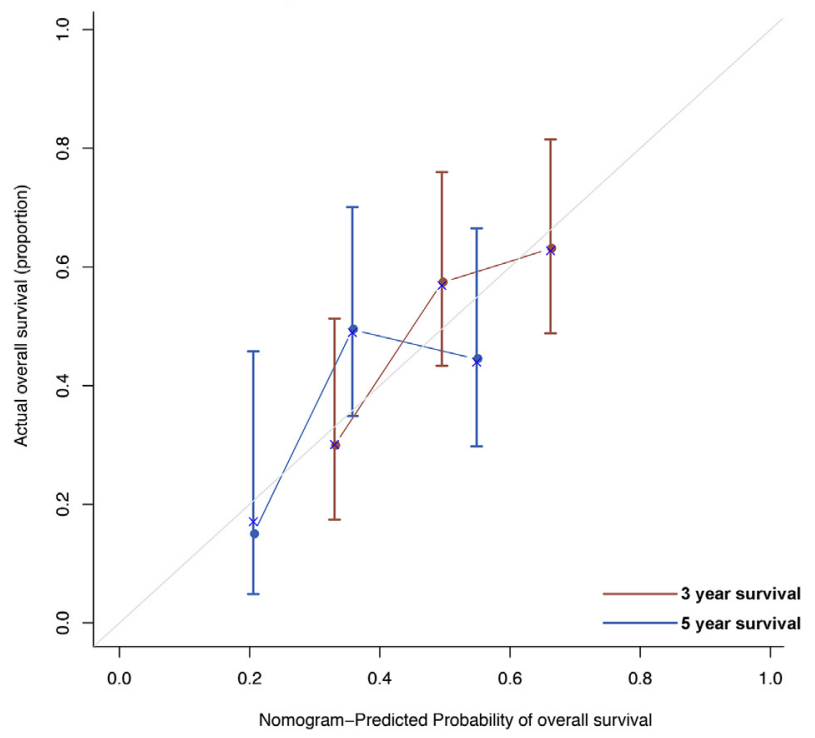

FIGURE 3. The calibration plot for the nomogram in the validation cohort. Nomogram-predicted survival is plotted on the $\mathrm{x}$-axis; observation survival is plotted on the $y$-axis. A plot along the 45-degree line indicated a perfect calibration model in which the predicted probabilities were consistent with the actual outcomes. The blue line represents 5-year survival. The red line represents 3-year survival.

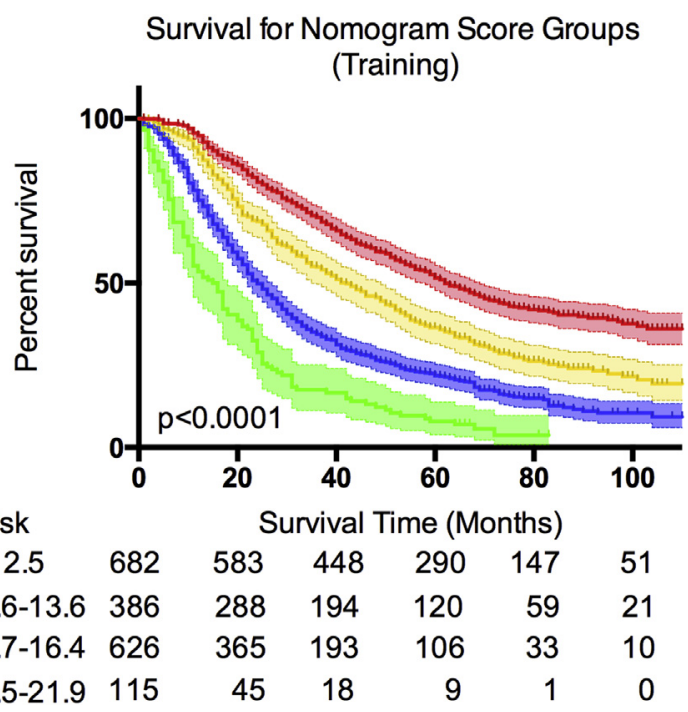

FIGURE 4. Kaplan-Meier survival curves of stratified risk groups in the training group. The number of patients at risk is listed under the figure. The dotted line represents the $95 \%$ confidence intervals.

model. Furthermore, this model was validated in an independent cohort of Chinese patients, which raised the generalizability of the model.

A comprehensive literature review identified that age, sex, and histology were common independent prognostic factors for locally advanced NSCLC and adopted by several published models. ${ }^{11,18,19}$ Similar results were observed in age and sex variables of our model. However, histology was not a significant predictor after multivariate cox analysis. The possible reason might be low frequency of

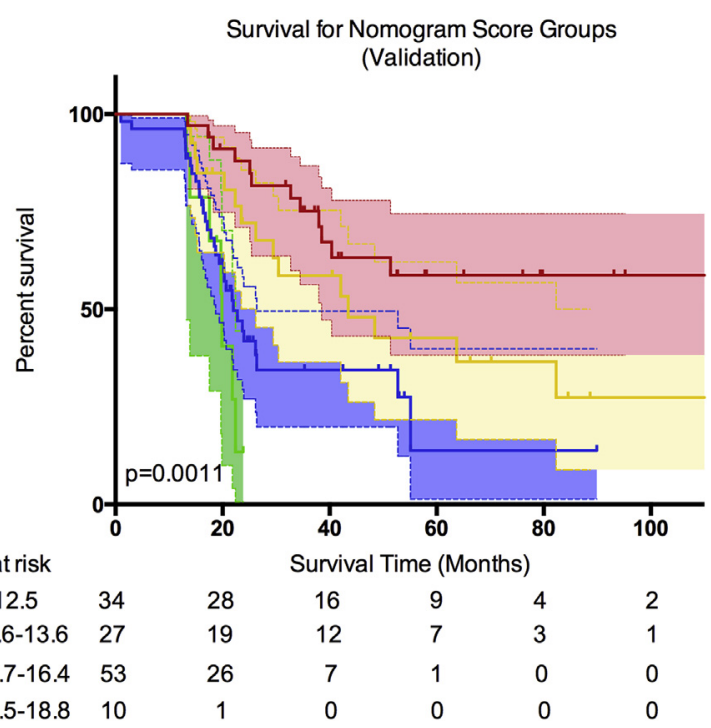

FIGURE 5. Kaplan-Meier survival curves of stratified risk groups in the validation group. The number of patients at risk is listed under the figure. The dotted line represents the $95 \%$ confidence intervals. 
TABLE 3. Kaplan-Meier survival analysis of stratified groups

\begin{tabular}{ccccc}
\hline Group & $\begin{array}{c}\text { 0-12.5 } \\
(\boldsymbol{P} \text { value })\end{array}$ & $\begin{array}{c}\text { 12.6-13.6 } \\
(\boldsymbol{P} \text { value })\end{array}$ & $\begin{array}{c}\mathbf{1 3 . 7 - 1 6 . 4} \\
(\boldsymbol{P} \text { value })\end{array}$ & $\begin{array}{c}\mathbf{1 6 . 5 - 2 1 . 9} \\
(\boldsymbol{P} \text { value })\end{array}$ \\
\hline Training & & & & \\
$0-12.5$ & - & $<0.001^{*}$ & $<0.001^{*}$ & $<0.001^{*}$ \\
$12.6-13.6$ & $<0.001^{*}$ & - & $<0.001^{*}$ & $<0.001^{*}$ \\
$13.7-16.4$ & $<0.001^{*}$ & $<0.001^{*}$ & - & $<0.001^{*}$ \\
16.5-21.9 & $<0.001^{*}$ & $<0.001^{*}$ & $<0.001^{*}$ & - \\
Validation & & & & \\
0-12.5 & - & 0.099 & $<0.001^{*}$ & $<0.001^{*}$ \\
$12.6-13.6$ & 0.099 & - & $0.050^{*}$ & $0.002^{*}$ \\
$13.7-16.4$ & $<0.001^{*}$ & $0.050^{*}$ & - & 0.227 \\
$16.5-21.9$ & $<0.001^{*}$ & $0.002^{*}$ & 0.227 & - \\
\hline
\end{tabular}

* Statistically significant.

large cell carcinoma in the training cohort. In light of the significance of histology reported by previous studies, we incorporated it into subsequent analyses. In addition, increasing evidence indicated that tumor size was an independent prognostic factor for node-positive and locally invasive disease. $^{20,21}$ Incorporating tumor size might improve the accuracy of OS prediction, ${ }^{21,22}$ which reinforced reliability of tumor size in our model. Furthermore, tumor grade reflected the differentiated ability and malignant degree of the cancer, and visceral pleural invasion assessed the aggressiveness of tumors. Both were significantly related to prognosis. ${ }^{18,23,24}$ However, few paper analyzed the prediction ability of visceral pleural invasion in locally advanced NSCLC. This study demonstrated that tumor grade and visceral pleural invasion were significant variables, which would improve the accuracy of survival prediction.

Adequate evaluation of the involved lymph nodes was critical for the postoperative survival of patients with stage IIIA-N2 NSCLC. ${ }^{25}$ It was clear from the recent literature that patients with more positive lymph nodes tended to have a worse prognosis compared with those with fewer ones. ${ }^{26}$ In addition, the number of lymph nodes examined was another important prognostic predictor for NSCLC. ${ }^{27}$ Recent studies indicate that the ratio of positive lymph nodes and lymph nodes examined is a significant prognostic factor for NSCLC. ${ }^{28}$ However, it was not appropriate for a nomogram and would potentially decrease the accuracy of the model. Because the ratio was the quotient that was highly dependent on adequacy of lymphadenectomy and pathologic review, ${ }^{29}$ which implied that 2 same ratio patients with different numbers of positive lymph nodes or different numbers of lymph nodes examined might have the same point and similar prognosis, we incorporated the number of positive lymph nodes and the number of lymph nodes examined as 2 continuous variables into the model, which preserved enough information on the lymph nodes and strengthened the accuracy of the nomogram.
It was necessary to validate and calibrate the nomogram to avoid overfitting. The calibration plot showed good consistency between the nomogram's predicted survival and actual observed survival, which indicated good reliability of the nomogram. Our nomogram discriminatory capability was only $67.3 \%$, which was considered weak. Because patients who received postoperative or preoperative therapies were included in the nomogram, the $\mathrm{C}$-index might be affected by the contradictory impact of postoperative or preoperative therapies. ${ }^{11}$ In addition, the validation cohort discriminatory capacity of $66.4 \%$ was similar to the training cohort at $67.3 \%$. Moreover, survival differences were observed between 4 risk subgroups stratified by the optimal cut-off score, indicating a good discrimination ability of the nomogram.

However, there were still some limitations in the current study. The major limitation was lack of chemotherapy information in the SEER database. The SEER MedicareLinked database only provided chemotherapy information for patients older than 65 years. Thus, we couldn't obtain how many patients receiving preoperative or postoperative chemotherapy in our training cohort, which might influence the performance of the model. In addition, we could not access more pretreatment variables related to survival. For example, comorbidity, forced expiratory volume in 1 second $\left(\mathrm{FEV}_{1}\right)$, diffusing capacity of the lungs for carbon monoxide (DLCO), and performance score were vital prognostic predictors for locally advanced NSCLC, which would make the model more meaningful and useful. Furthermore, the information for upstaged and downstages of patients who received preoperative induction therapy was unknown. Increasing evidence indicated that downstages of the mediastinal nodes were correlated with better prognosis. ${ }^{30,31}$ It was important to note that our analysis excluded $15 \%$ of patients from the initial group due to missing lymph node data in SEER, which would result in potential limitations in generalizability. Thus, we compared the distribution of baseline variables in excluded patients compared with the included patients and the survival in the excluded versus included patients. The results indicated that no significant difference was observed between the exclusion group and the inclusion group. In current study, we provided a feasible model to individually predict the survival (Figure E2 and Table E2), which might benefit survival counseling for patients and clinicians, clinical trial design and follow-up, as well as postoperative strategy-making.

\section{Conflict of Interest Statement}

Authors have nothing to disclose with regard to commercial support.

\footnotetext{
References

1. Gillaspie EA, Wigle DA. Management of stage IIIA (N2) non-small cell lung cancer. Thorac Surg Clin. 2016;26:271-85.
} 
2. Thomas M, Rübe C, Hoffknecht P, Macha HN, Freitag L, Linder A, et al. Effect of preoperative chemoradiation in addition to preoperative chemotherapy: a randomised trial in stage III non-small-cell lung cancer. Lancet Oncol. 2008;9; 636-48.

3. Vansteenkiste J, Crino L, Dooms C, Douillard JY, Faivre-Finn C, Lim E, et al. 2nd ESMO Consensus Conference on Lung Cancer: early-stage non-small-cell lung cancer consensus on diagnosis, treatment and follow-up. Ann Oncol. 2014;25:1462-74.

4. Eberhardt WEE, De Ruysscher D, Weder W, Le Péchoux C, De Leyn P, Hoffmann H, et al. 2nd ESMO Consensus Conference in Lung Cancer: locally advanced stage III non-small-cell lung cancer. Ann Oncol. 2015;26:1573-88.

5. Andre F, Grunenwald D, Pignon J-P, Dujon A, Pujol JL, Brichon PY, et al. Survival of patients with resected N2 non-small-cell lung cancer: evidence for a subclassification and implications. J Clin Oncol. 2000;18:2981-9.

6. Decaluwé H, De Leyn P, Vansteenkiste J, Dooms C, Van Raemdonck D, Nafteux P, et al. Surgical multimodality treatment for baseline resectable stage IIIA-N2 non-small cell lung cancer. Degree of mediastinal lymph node involvement and impact on survival. Eur J Cardiothorac Surg. 2009;36:433-9.

7. Dickhoff C, Hartemink KJ, Kooij J, van de Ven PM, Paul MA, Smit EF, et al. Is the routine use of trimodality therapy for selected patients with non-small cell lung cancer supported by long-term clinical outcomes? Ann Oncol. 2017;28:185.

8. Eberhardt WEE, Pöttgen C, Gauler TC, Friedel G, Veit S, Heinrich V, et al. Phase III Study of Surgery Versus Definitive Concurrent Chemoradiotherapy Boost in Patients With Resectable Stage IIIA(N2) and Selected IIIB Non-Small-Cell Lung Cancer After Induction Chemotherapy and Concurrent Chemoradiotherapy (ESPATUE). J Clin Oncol. 2015;33:4194-201.

9. Iasonos A, Schrag D, Raj GV, Panageas KS. How to build and interpret a nomogram for cancer prognosis. J Clin Oncol. 2008;26:1364-70.

10. Wang Y, Li J, Xia Y, Wang K, Yan Z, Wan X, et al. Prognostic nomogram for intrahepatic cholangiocarcinoma after partial hepatectomy. J Clin Oncol. 2013;31: 1188-95.

11. Liang W, Zhang L, Jiang G, Wang Q, Liu L, Liu D, et al. Development and validation of a nomogram for predicting survival in patients with resected non-smallcell lung cancer. J Clin Oncol. 2015;33:861-9.

12. Zhang Y, Sun Y, Xiang J, Zhang Y, Hu H, Chen H. A clinicopathologic prediction model for postoperative recurrence in stage Ia non-small cell lung cancer. $J$ Thorac Cardiovasc Surg. 2014;148:1193-9.

13. Attaar A, Winger DG, Luketich JD, Schuchert MJ, Sarkaria IS, Christie NA, Nason KS. A clinical prediction model for prolonged air leak after pulmonary resection. J Thorac Cardiovasc Surg. 2017;153:690-9.e692.

14. Balachandran VP, Gonen M, Smith JJ, DeMatteo RP. Nomograms in oncology: more than meets the eye. Lancet Oncol. 2015;16:e173-80.

15. Harrell FE Jr, Califf RM, Pryor DB, Lee KL, Rosati RA. Evaluating the yield of medical tests. JAMA. 1982;247:2543-6.

16. Camp RL, Dolled-Filhart M, Rimm DL. X-tile: a new bio-informatics tool for biomarker assessment and outcome-based cut-point optimization. Clin Cancer Res. 2004;10:7252-9.

17. Jin C, Cao J, Cai Y, Wang L, Liu K, Shen W, Hu J. A nomogram for predicting the risk of invasive pulmonary adenocarcinoma for patients with solitary peripheral subsolid nodules. J Thorac Cardiovasc Surg. 2017;153:462-9.e461.
18. Oberije C, De Ruysscher D, Houben R, van de Heuvel M, Uyterlinde W, Deasy JO, et al. A validated prediction model for overall survival from stage III non-small cell lung cancer: toward survival prediction for individual patients. Int J Radiat Oncol Biol Phys. 2015;92:935-44.

19. Rocco G, Nason K, Brunelli A, Varela G, Waddell T, Jones DR. Management of stage IIIA (N2) non-small cell lung cancer: a transatlantic perspective. J Thorac Cardiovasc Surg. 2016;151:1235-8.

20. Cho S, Kim K, Jheon S. Characteristics and prognostic factors of node-negative non-small cell lung cancer larger than $5 \mathrm{~cm}$. Interact Cardiovasc Thorac Surg. 2017;25:448-54.

21. Zhang J, Gold KA, Lin HY, Swisher SG, Xing Y, Lee JJ, et al. Relationship between tumor size and survival in non-small-cell lung cancer (NSCLC): an analysis of the Surveillance, Epidemiology, and End Results (SEER) registry. J Thorac Oncol. 2015;10:682-90.

22. Morgensztern D, Waqar S, Subramanian J, Gao F, Trinkaus K, Govindan R. Prognostic significance of tumor size in patients with stage III non-small-cell lung cancer: a Surveillance, Epidemiology, and End Results (SEER) survey from 1998 to 2003. J Thorac Oncol. 2012;7:1479-84.

23. Sun Z, Aubry M-C, Deschamps C, Marks RS, Okuno SH, Williams BA, et al. Histologic grade is an independent prognostic factor for survival in non-small cell lung cancer: an analysis of 5018 hospital- and 712 population-based cases. J Thorac Cardiovasc Surg. 2006;131:1014-20.

24. Tian D, Pei Y, Zheng Q, Zhang J, Li S, Wang X, et al. Effect of visceral pleural invasion on the prognosis of patients with lymph node negative non-small cell lung cancer. Thorac Cancer. 2017;8:97-105.

25. Zhang Y, Sun Y, Chen H. Effect of tumor size on prognosis of node-negative lung cancer with sufficient lymph node examination and no disease extension. Onco Targets Ther. 2016;9:649-53.

26. Zhao Y, Li G, Zheng D, Jia M, Dai W, Sun Y, Chen H. The prognostic value of lymph node ratio and log odds of positive lymph nodes in patients with lung adenocarcinoma. J Thorac Cardiovasc Surg. 2017;153:702-9.e701.

27. Wang S, Zhang B, Li C, Cui C, Yue D, Shi B, et al. Prognostic value of number of negative lymph node in patients with stage II and IIIa non-small cell lung cancer. Oncotarget. 2017;8:79387-96.

28. Taylor MD, LaPar DJ, Thomas CJ, Persinger M, Stelow EB, Kozower BD, et al. Lymph node ratio predicts recurrence and survival after R0 resection for nonsmall cell lung cancer. Ann Thorac Surg. 2013;96:1163-70.

29. Rice TW, Blackstone EH. Lymph node ratio: a confounded quotient. Ann Thorac Surg. 2013;96:744.

30. Ziel E, Hermann G, Sen N, Bonomi P, Liptay MJ, Fidler MJ, et al. survival benefit of surgery after chemoradiotherapy for stage III (N0-2) non-small-cell lung cancer is dependent on pathologic nodal response. J Thorac Oncol. 2015;10: 1475-80.

31. Jeremić B, Casas F, Dubinsky P, Gomez-Caamano A, Čihorić N, Videtic G. Surgery for stage IIIa non-small-cell lung cancer: lack of predictive and prognostic factors identifying any subgroup of patients benefiting from It. Clin Lung Cancer. 2016;17:107-12.

Key Words: nomogram, stage IIIA-N2 NSCLC, surgery, prognosis 


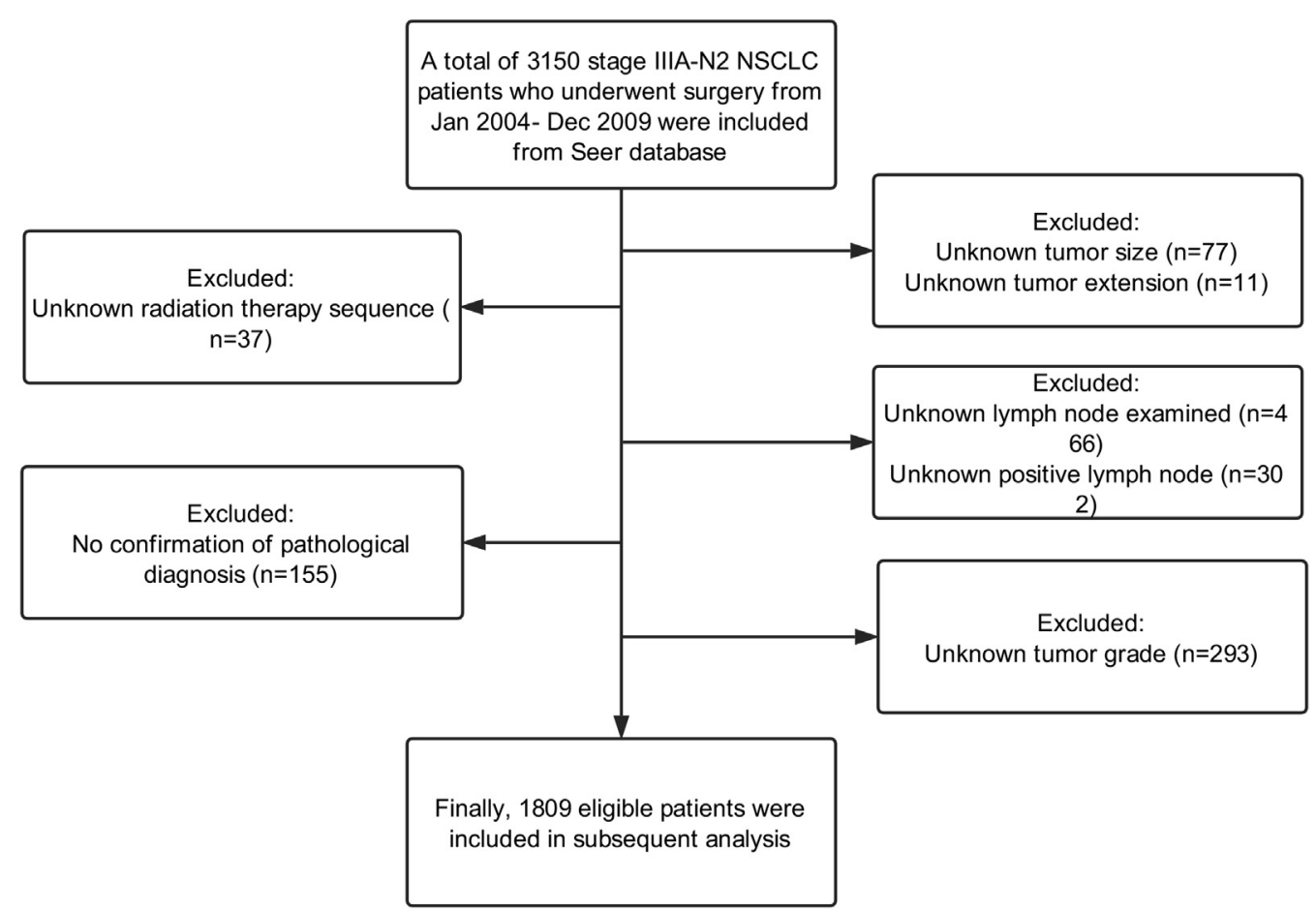

FIGURE E1. The consort diagram showing the inclusion and exclusion of patients. NSCLC, Non-small cell lung cancer.

\section{Inclusion vs Exclusion group}

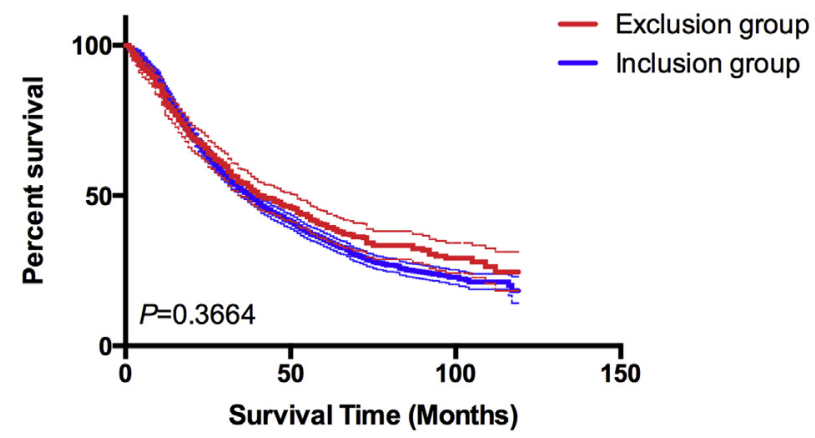

FIGURE E2. Comparison of the survival between the inclusion subgroup and the exclusion subgroup. The dotted line represents the $95 \%$ confidence intervals. 
TABLE E1. Coefficients of each variables

\begin{tabular}{|c|c|}
\hline Variables & Coefficients \\
\hline Age & 0.0205 \\
\hline Sex & 0.2369 \\
\hline \multicolumn{2}{|l|}{ Histology } \\
\hline Adenocarcinoma & 0 \\
\hline Large cell carcinoma & 0.0914 \\
\hline Squamous cell carcinoma & 0.3335 \\
\hline \multicolumn{2}{|l|}{ Grade } \\
\hline Well-differentiated & 0 \\
\hline Moderately differentiated & 0.3326 \\
\hline Poorly differentiated & 0.3747 \\
\hline Undifferentiated & 0.5212 \\
\hline Lymph nodes examined, number & -0.0360 \\
\hline Positive lymph nodes, number & 0.1013 \\
\hline Tumor size & 0.0082 \\
\hline Visceral pleural invasion & 0.2314 \\
\hline Surgery type & 0.0221 \\
\hline
\end{tabular}


TABLE E2. Comparison of inclusion group and exclusion group

\begin{tabular}{|c|c|c|c|}
\hline Demographic & Inclusion group & Exclusion group & $P$ value \\
\hline Total & 1809 & 466 & \\
\hline Age, y, median (IQR) & $65(57.5-72.5)$ & $64(57-70)$ & .098 \\
\hline \multicolumn{4}{|l|}{ Sex } \\
\hline Female & 950 & 229 & .212 \\
\hline Male & 859 & 237 & \\
\hline \multicolumn{4}{|l|}{ Tumor site } \\
\hline Main bronchus & 22 & 9 & .383 \\
\hline Upper lobe & 1043 & 281 & \\
\hline Middle lobe & 84 & 25 & \\
\hline Lower lobe & 602 & 134 & \\
\hline Overlap lobe & 39 & 13 & \\
\hline Lung, NOS & 19 & 4 & \\
\hline \multicolumn{4}{|l|}{ Histology } \\
\hline Adenocarcinoma & 1294 & 310 & .111 \\
\hline Lung squamous cell carcinoma & 444 & 135 & \\
\hline Large cell carcinoma & 71 & 21 & \\
\hline \multicolumn{4}{|l|}{ Grade } \\
\hline Well-differentiated & 96 & 32 & .504 \\
\hline Moderately differentiated & 803 & 196 & \\
\hline Poorly differentiated & 847 & 219 & \\
\hline Undifferentiated & 63 & 19 & \\
\hline \multicolumn{4}{|l|}{ Number lymph nodes examined } \\
\hline Median & 10 & - & - \\
\hline IQR & $6-15$ & - & \\
\hline \multicolumn{4}{|l|}{ Number of positive lymph node } \\
\hline Median & 3 & - & - \\
\hline IQR & $1-5$ & - & \\
\hline \multicolumn{4}{|l|}{ Tumor size, mm } \\
\hline Median & 34 & 37 & .103 \\
\hline IQR & $23-49$ & $25-60$ & \\
\hline \multicolumn{4}{|l|}{ Surgery type } \\
\hline Lobectomy & 1602 & 401 & .260 \\
\hline pneumonectomy & 207 & 65 & \\
\hline \multicolumn{4}{|l|}{ Therapy } \\
\hline Surgery only & 1139 & 278 & .409 \\
\hline Radiation after surgery & 558 & 155 & \\
\hline Radiation before surgery & 112 & 33 & \\
\hline \multicolumn{4}{|l|}{ Visceral pleural invasion } \\
\hline No & 879 & 212 & .254 \\
\hline Yes & 930 & 254 & \\
\hline
\end{tabular}

$I Q R$, Interquartile; $N O S$, not otherwise specified. 